\title{
SCOTTY CRAMP - A CASE REPORT
}

\section{FABIANO MONTIANI FERREIRA}

Professor of Internal Medicine, Departament of Veterinary Medicine, Federal University of Paraná

The so-called Scotty Cramp is an inherited paroxysmal neurological disorder with a recessive mode of transmission in Scottish terrier dogs. Pharmacological studies suggest that the disorder is associated with serotonergic transmitters imbalances, causing severe muscular hypertonicity episodes. The objective of this work is to report a case evolving a 3 year-old, male Scottish Terrier, named Joca, weighting $20 \mathrm{~kg}$, admitted to the UFPR Animal Hospital. The owners reported that the patient was experiencing sporadical episodes of pelvic limb muscle stiffness and intense vocalization, as if he was suffering from an intense pain condition. On clinical and radiographic examination the patient had no abnormalities. At the end of the visit he suddenly was arching the lumbar spine with the tail and pelvic limbs tightly flexed against the body, this position was followed by a transient apnea episode and a loud scream. The patient then started to whine for approximately 2 minutes. There was no apparent site of localized pain on palpation. The owners reported that this episode was very similar to what they have seen at home. Laboratory tests results were: alkaline phosphatase (ALP)- $67,8 \mathrm{IU} / \mathrm{L}$, creatinine- $0,82 \mathrm{mg} / \mathrm{dl}$, alanine aminotransferase (ALT)- 35,5 IU/L creatine phosphokinase (CPK)- $279 \mathrm{IU}$, revealing a very mild increase on CPK value. Diagnosis was Scotty Cramp based on history, clinical signs and tests results. The treatment protocol was diazepam $0,25 \mathrm{mg} / \mathrm{kg}$ every 24 hours and vitamin E $125 \mathrm{IU} / \mathrm{kg}$ every 24 hours as well. After two weeks of treatment the patient had another episode of muscle cramping and then never had any episodes again, until now. 